\title{
Formulation of a Self-Consistent Model for Quantum Well pin Solar Cells: Dark Behavior
}

\author{
S. RAMEY and R. KHOIE* \\ Department of Electrical and Computer Engineering, University of Nevada, Las Vegas, 89154
}

(Received 28 May 1997; In final form 15 July 1997)

\begin{abstract}
A self-consistent numerical simulation model for a pin single-cell solar cell is formulated. The solar cell device consists of a $p-\mathrm{AlGaAs}$ region, an intrinsic $i-\mathrm{AlGaAs} / \mathrm{GaAs}$ region with several quantum wells, and a $n-\mathrm{AlGaAs}$ region. Our simulator solves a field-dependent Schrödinger equation self-consistently with Poisson and drift-diffusion equations. The field-dependent Schrödinger equation is solved using the transfer matrix method. The eigenfunctions and eigenenergies obtained are used to calculate the escape rate of carriers from the quantum wells, the capture rates of carriers by the wells, the absorption spectra in the wells, and the non-radiative recombination rates of carriers in the quantum wells. These rates are then used in a self-consistent finite-difference numerical Poisson-drift-diffusion solver. We believe this is the first such comprehensive model ever reported.
\end{abstract}

Keywords: Quantum well, solar cell, Schrödinger, escape, capture, recombination

\section{INTRODUCTION}

The conversion efficiency of a single cell pin solar cell can be enhanced by incorporating quantum wells in the intrinsic region of the device. [1] The incorporation of the quantum wells has two counteracting effects: the short-circuit current is increased because of the additional absorption of the low-energy photons in the lower bandgap quantum well and the open-circuit voltage is decreased because of the increase in the recombination of the photoexcited carriers trapped in the quantum well. Experimental results have shown, nevertheless, that the additional photocurrent resulting from the extension of the absorption spectrum to lower energies can outweigh the accompanying drop in the open-circuit voltage [2-3].

Along with these experimental studies, a number of theoretical investigations have been performed. Corkish and Green [4] studied the effects of recombination of carriers in the quantum well and concluded that although the increased recombination reduces the open-circuit voltage, limited

\footnotetext{
* Corresponding author.
} 
enhancement in the conversion efficiency can be obtained with incorporation of the quantum well, albeit not as much as previously reported by Barnham and Duggan [1]. Araujo et al. [5] used detailed balance theory and predicted that the conversion efficiency of the quantum well cell would not exceed that of the base-line device. The results of photoresponse calculations by Renaud et al. [6] revealed that introducing the quantum wells can lead to improved photocurrent without much degradation of the open-circuit voltage. Most recently, Anderson [7] presented an ideal model for the quantum well solar cell device, incorporating the recombination and generation in the quantum wells. Anderson concluded that the improvement in efficiency is achieved only when the depth of the quantum well is less than about $200 \mathrm{meV}$.

The need for a comprehensive model is rather obvious, now that there seems to be an unsolved debate as to the ultimate advantage of incorporating quantum wells in the intrinsic region of a pin solar cell. In this paper we present formulation of one such model in which we self-consistently include the effects of:

1) Capture of electrons by the wells,

2) Escape of electrons from the wells,

3) Absorption of light in the wells, and

4) Recombination of carriers in the wells.

The standard drift-diffusion equations are modified to account for generation and recombination in the quantum wells and the transfer of electrons and holes between the bulk and quantum well systems.

\section{SELF-CONSISTENT MODEL}

The steady-state transport of carriers in the pin structure is described by current continuity equation written for the bulk regions as:

$$
\frac{\partial n_{b}}{\partial t}=G_{b}-U_{b}+\frac{n_{w}}{\tau_{e}^{n}}-\frac{n_{b}}{\tau_{c}^{n}}+\frac{1}{q} \frac{d J_{n}}{d x}=0,
$$

where $n_{b}$ and $n_{w}$ are electron densities in the bulk and quantum wells, respectively. $J_{n}$ is electron current density. The two additional terms to the standard bulk current continuity equation incorporate the effect of carrier transfer into and out of the quantum wells. The terms $n_{w} / \tau_{e}^{n}$ and $n_{b} / \tau_{c}^{n}$ are the electrons escape and capture rates, respectively. These rates are also used in the continuity equations for the quantum wells, as given by:

$$
\frac{\partial n_{w}}{\partial t}=\frac{n_{b}}{\tau_{c}^{n}}-\frac{n_{w}}{\tau_{e}^{n}}+G_{w}-U_{w}=0 .
$$

In the above equations $\tau_{e}^{n}$ and $\tau_{c}^{n}$ are the electrons escape and capture times, respectively. The escape times of carriers are calculated using the model reported by Moss et al. [8] and capture times are extrapolated from theoretical and experimental data reported by Blom et al. [9]. Similar equations are written for holes. The boundary conditions for continuity equations are derived from surface recombination velocity model.

In the above continuity equations, the recombination in the bulk is modeled with radiative and non-radiative mechanisms. The term $G_{b}$ is bulk generation rate and is given by:

$$
G_{b}=\int_{0}^{\lambda_{c}} \alpha(\lambda) \cdot N_{p h} \cdot\left[\exp \left(-\int_{0}^{x} \alpha(\lambda) d x\right)\right] d \lambda
$$

where $\lambda_{c}$ is set to correspond to the bandgap of the material. The recombination in the quantum well $U_{w}$ is a modified Shockley-Read-Hall recombination rate given by:

$$
U_{w}=\frac{\sigma_{n} \sigma_{p} v_{t h} N_{t}\left[p n-p_{0} n_{0}\right]}{\sigma_{n}\left[n+n_{t}\right]+\sigma_{p}\left[p+p_{t}\right]}
$$

where the trap density, $N_{t}$, is derived from the density of the interface states. The generation term $G_{w}$ is calculated from Eq. (3) with the bulk absorption coefficient replaced with that of the quantum well. The absorption coefficients of the quantum wells are calculated by a model reported by Stevens et al. [10]. 
Calculation of the absorption spectra in the quantum wells as well as the escape and capture times of carriers require the eigenfunctions and eigenenergies of the carriers in the quantum wells, which are obtained from a field-dependent Schrödinger equation given by:

$$
\left[\frac{\hbar^{2}}{2} \frac{d}{d x} \frac{1}{m^{*}(x)} \frac{d}{d x}+V(x)\right] \psi(x)=E_{i} \psi(x)
$$

where $\psi(x)$ is the envelope function, $E_{i}$ are the eigenenergies and $V(x)$ is the potential profile. Non-constant effective mass $m^{*}(x)$ is assumed. The Schrödinger equation is solved using the transfer matrix method [11]. The above equations, together with Poisson and drift-diffusion equations are solved using a finite difference scheme.

\section{RESULTS}

The energy band diagram of the pin solar cell device with four quantum wells in the intrinsic region is shown in Figure 1. We simulated five pin devices all with a acceptor doping level of $10^{18} / \mathrm{cc}$

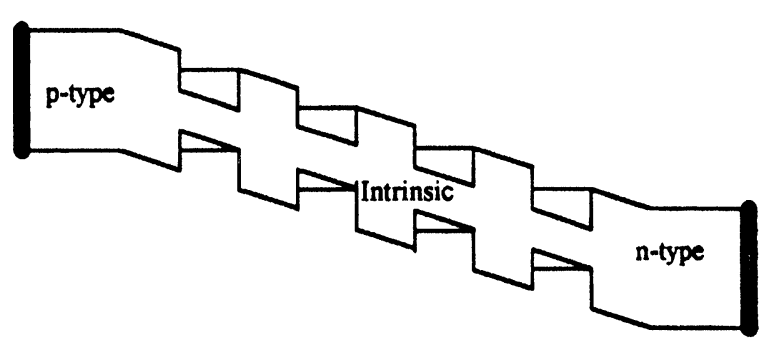

FIGURE 1 Energy band diagram of the solar cell with four quantum wells.

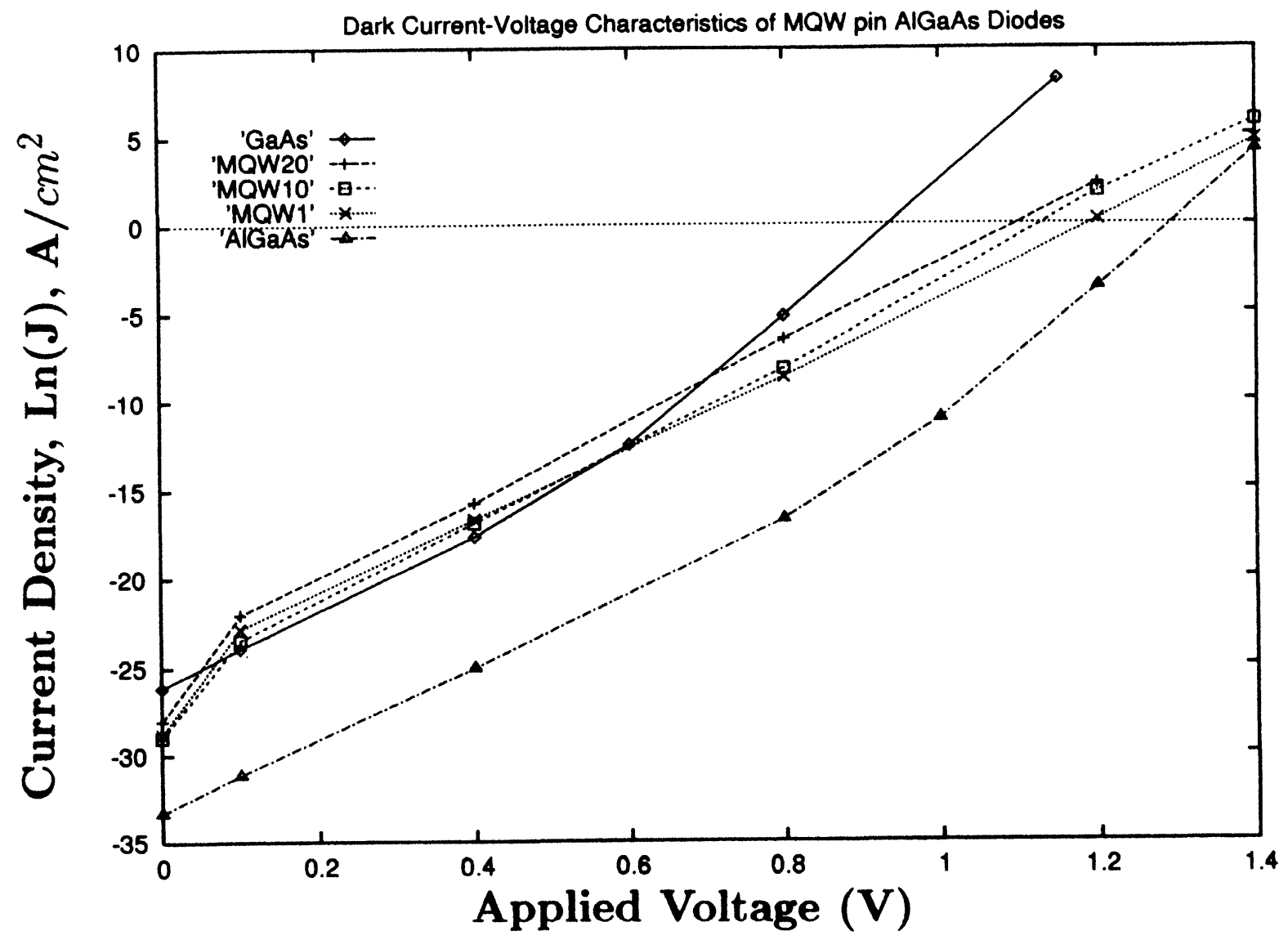

FIGURE 2 Dark characteristics of MQW pin solar cells. 
and donor doping level of $4 \times 10^{17} / \mathrm{cc}$ in the 0.2 $\mu \mathrm{m} p$-region and $0.3 \mu \mathrm{m} n$-region, respectively. The intrinsic region of all five devices is $0.5 \mu \mathrm{m}$. The first device is a base-line GaAs, the second device is a base-line $\mathrm{AlGaAs}$ device and the remaining three devices are $\mathrm{AlGaAs}$ devices with 1,10 and 20 quantum wells, respectively. The mole fraction of $\mathrm{Al}$ is 0.3 in all $\mathrm{AlGaAs}$ devices and all quantum wells are $100 \AA$ 为 wide.

We simulated the dark characteristics of all five cells and the results are shown in Figure 2. Because the model incorporates both radiative and nonradiative recombination for the bulk material, the slope of the dark current is $q v / n k T$, with the ideality factor $n$ ranging from one for exclusively radiative recombination to two for exclusively non-radiative. In the pin devices without quantum wells, $n$ approaches one at high forward bias where radiative recombination becomes more important. At low biases the non-radiative recombination dominates and $n$ is 1.9 for the $\mathrm{AlGaAs}$ device at 0.1 volts applied.

The recombination in the quantum wells is modeled as non-radiative interface recombination using a modified Shockley-Read-Hall expression. With the introduction of quantum wells into the pin device, this non-radiative recombination dominates at all biases and the ideality factor equals about 1.7 for all the quantum well devices. The observed crossover of the $i-v$ curves of the quantum well devices and the GaAs device has also been reported by Ragay et al. [3].

\section{References}

[1] Barnham, K. and Duggan, J. (1990). "A new approach to high-efficiency multi-band-gap solar cells", Journal of Applied Physics, 67, 3490-3493.

[2] Barnham, K., Braun, B., Nelson, J. and Paxman, M. (1991). "Short-circuit current and energy efficiency enhancement in a low-dimensional structure photovoltaic device", Applied Physics Letters, 59, 135-137.

[3] Ragay, F., Wolter, J., Marti, A. and Araujo, G. (1994) "Experimental analysis of the efficiency of MQW solar cells", Proceedings of the 12th European Community Photovoltaic Solar Energy Conference, pp. 1429-1433.

[4] Corkish, R. and Green, M. (1993). "Recombination of carriers in quantum well solar cells", Proceedings of the 23rd Photovoltaics Specialist Conference, pp. 675-680.
[5] Araujo, G., Marti, A., Ragay, F. and Wolter, J. (1994). "Efficiency of multiple quantum well solar cells", Proceedings of the 12th European Community Photovoltaic Solar Energy Conference, pp. 1435-1439.

[6] Renaud, P., Vilela, M., Freundlich, A., Bensaoula, A. and Medelci, N. (1995). "Modeling $p-i$ (MQW)- $n$ solar cells: A contribution for a near optimum design", Proceedings of the 1994 IEEE First World Conference on Photovoltaic Energy Conversion Hawaii, IEEE, NY, pp. 1787-1790.

[7] Anderson, N. (1995). "Ideal Theory of Quantum Well Solar Cells", Journal of Applied Physics, 78, 1850-1861.

[8] Moss, D., Ido, T. and Sano, H. (1994). "Calculation of photo-generated carrier escape rates from GaAs/AlGaAs quantum wells", IEEE Journal of Quantum Electronics, 30, $1015-1026$.

[9] Blom, P., Smit, C., Haverkort, J. and Wolter, J. (1993). "Carrier capture in a semiconductor quantum well", Physical Review B, 47, 2072-2081.

[10] Stevens, P., Whitehead, M., Parry, G. and Woodbridge, K. (1988). "Computer modeling of the electric field dependent absorption spectrum of multiple quantum well material", IEEE Journal Quantum Electronics, 24, 2007-2015.

[11] Jonsson, B. and Eng, S. (1990). "Solving the Schrodinger equation in arbitrary quantum-well potential profiles using the transfer matrix method", IEEE Journal of Quantum Electronics, 26, 2025-2035.

\section{Authors' Biographies}

Steve Ramey received his B.S. in Physics from Carnegie Mellon University in 1992. $\mathrm{He}$ is currently completing his M.S. degree in Electrical Engineering at University of Nevada, Las Vegas. His research interests include the optoelectronic properties of quantum well structures, numerical modeling of semiconductor devices and applications of parallel supercomputers. He has accepted a research position with Solid State Measurements in Pittsburgh, Pennsylvania exploring the physics of metal-semiconductor contacts.

Rahim Khoie received his Ph.D. degree in Electrical Engineering from University of Pittsburgh, Pennsylvania, in 1986. He is presently an Associate Professor of Electrical and Computer Engineering at University of Nevada, Las Vegas. $\mathrm{He}$ has published numerous papers in journals and proceedings and has received several research grants. His research interest include high speed electron devices, heterostructures, quantum well devices, photovoltaics and optoelectronics. Dr. Khoie has won a number of local and national teaching awards. 

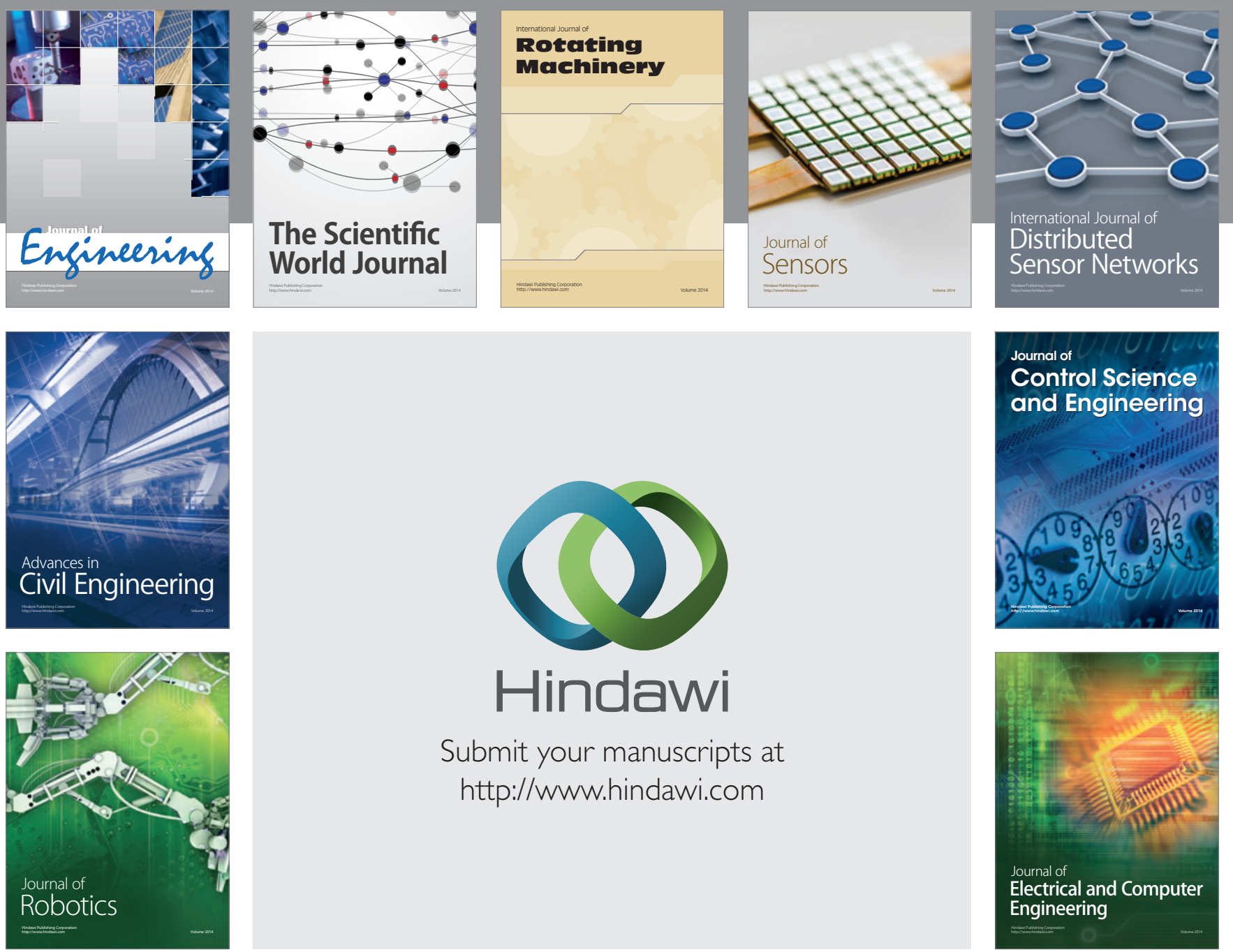

Submit your manuscripts at

http://www.hindawi.com
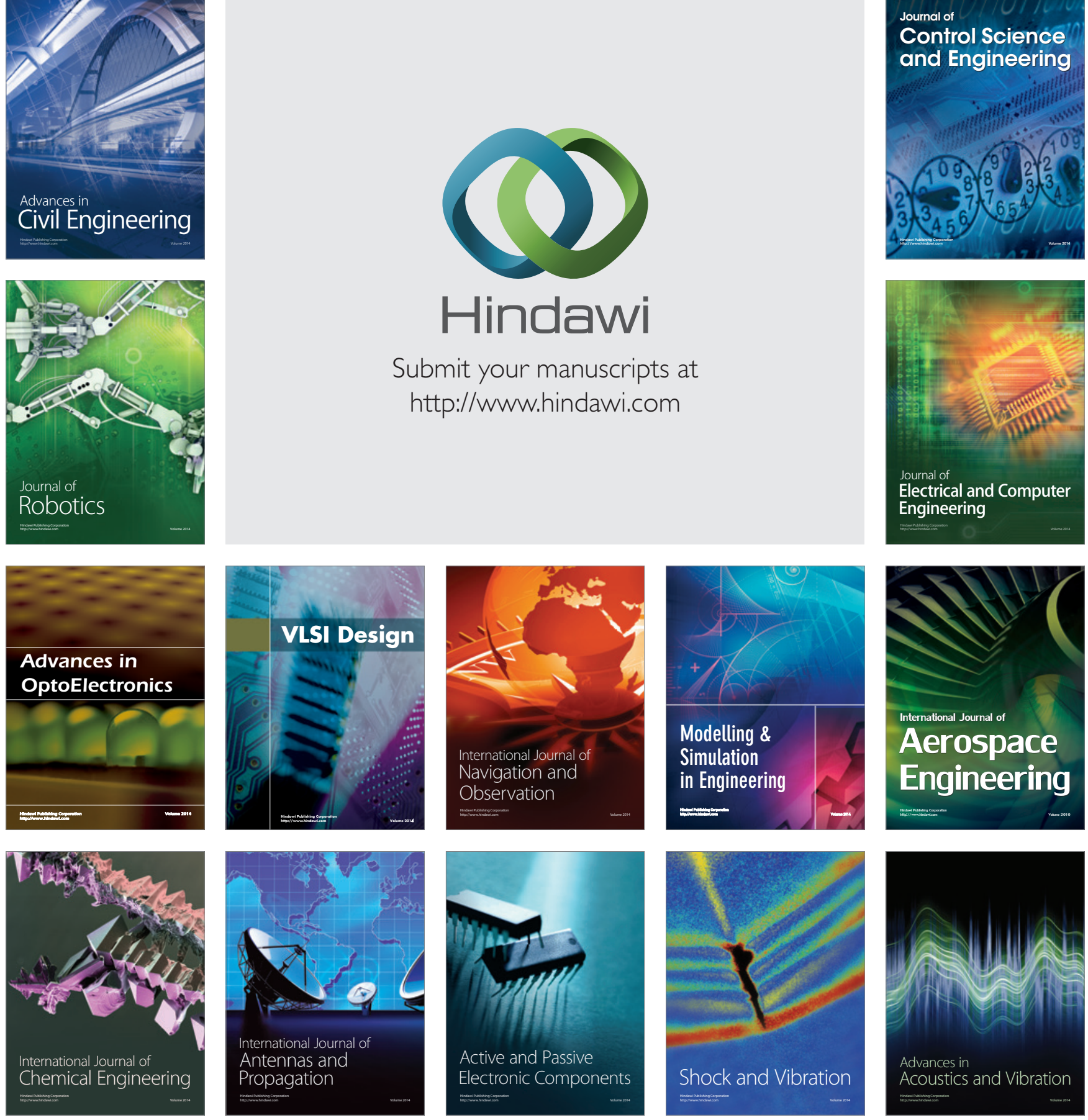